\title{
DESARROLLO EMOCIONAL EN LA INFANCIA. UN ESTUDIO SOBRE LAS COMPETENCIAS EMOCIONALES DE NIÑOS Y NIÑAS
}

\author{
Davinia Heras Sevilla \\ Amaya Cepa Serrano \\ Fernando Lara Ortega \\ Universidad de Burgos (España) \\ dheras@ubu.es \\ http://dx.doi.org/10.17060/ijodaep.2016.n1.v1.217
}

Fecha de Recepción: 8 Enero 2016

Fecha de Admisión: 15 Febrero 2016

\section{RESUMEN}

Este estudio tiene como objetivo la evaluación del desarrollo emocional de alumnado de Educación Infantil escolarizado en $2^{\circ}$ Curso del Segundo Ciclo de esta etapa $(\mathrm{N}=123)$. Para este fin, se han analizado las competencias emocionales de los menores. Concretamente, la conciencia emocional, la regulación emocional, la competencia social y las habilidades de vida para el bienestar. Además, se han comparado los resultados obtenidos por los niños y por las niñas en cada dimensión, a fin de conocer la influencia del sexo del menor en su desarrollo emocional.

Los resultados muestran diferencias significativas en función de la variable sexo en el nivel global de competencia emocional y en tres de las cuatro dimensiones que lo componen. El estudio evidencia además, la necesidad de evaluar los procesos emocionales en la infancia para favorecer una mejora en las competencias socio-emocionales del alumnado de Educación Infantil e incrementarlas a lo largo de su formación académica para su desarrollo integral.

Palabras claves: Desarrollo Emocional; Competencias Emocionales; Infancia; sexo.

\section{ABSTRACT}

This research has the purpose of evaluating emotional development in the student body during pre-school education in the second course of the second cycle of this stage $(\mathrm{N}=123)$.

To this end, children emotional competences have been analyzed. Specifically, emotional awareness, emotional regulation, social competence and life skills for welfare. Furthermore, the results obtained by little girls and boys have been compared in each dimension, with the aim of recognizing the influence of the sex of the infant during his emotional management.

The results show significant differences depending on the sex of the children in the global level of emotional competence and in three of the four dimensions which it is composed. Moreover, the study evidences the necessity of evaluating emotional processes during the infancy with the objec- 
tive of improving social and emotional competences of the student body of Pre-school education and increasing them throughout their academic training for their development.

Keywords: Emotional development; Emotional skills; Childhood; sex.

\section{ANTECEDENTES}

En los últimos años, las emociones y su aprendizaje a lo largo de la vida, han sido objeto de investigación; al igual que los estudios sobre cómo trabajarlas en el aula (Agulló, Filella, Soldevila, y Ribes, 2011; Ambrona, López-Pérez y Márquez-González, 2012; Fernandez-Berrocal y Extremera, 2009; Salovey y Grewal; 2005; Teruel, 2014).

Desde el nacimiento, niños y niñas se desarrollan en un ambiente impregnado de emociones. De esta manera, durante los primeros años de vida, el menor aprenderá a manifestar sus propias emociones, a recibir las de los demás, y a responder ante ellas tratando de controlar sus propias emociones. La forma en cómo realice este aprendizaje y en cómo se desarrolle emocionalmente dependerá su bienestar y calidad de vida.

Los primeros vínculos emocionales se establecen en el seno de la familia. La escuela supondrá el segundo agente educativo para el desarrollo emocional de los menores. En dicho contexto, resulta primordial potenciar y favorecer el desarrollo integral de todo el alumnado.

Tradicionalmente el sistema educativo se ha centrado en el desarrollo cognitivo. Como ya hemos indicado, en los últimos años se ha puesto de relieve la necesidad de trascender a este enfoque, trabajando desde una perspectiva más amplia que permita la inclusión de las emociones en al aula. Actualmente, no existe en el currículum escolar ninguna asignatura que trabaje explícitamente las competencias emocionales. Sin embargo, de una marea implícita, se les exige a los docentes que sus alumnos desarrollen sus capacidades afectivas.

Centrándonos en Educación Infantil, en la comunidad de Castilla y León, por ejemplo, en el Decreto 122/2007, de 27 de diciembre, se establece en su artículo tercero que la finalidad de la educación es contribuir al desarrollo físico, afectivo, social e intelectual de los niños. En este sentido, la legislación vigente reconoce la importancia del desarrollo afectivo y emocional para alcanzar un desarrollo integral de la personalidad.

Las corrientes científicas y teóricas actuales subrayan la necesidad de potenciar las competencias emocionales en Educación Infantil, a fin de favorecer el desarrollo emocional del menor (Dobrin y Kallay, 2013, Lau y Wu, 2012). Por ello, se considera fundamental que la educación obligatoria promueva el desarrollo de la competencia emocional; entendida como constructo complejo formado por diferentes dimensiones o habilidades. Para Salovey y Sluyter (1997, p 11) son cinco las dimensiones básicas que componen la competencia emocional: cooperación, asertividad, responsabilidad, empatía y autocontrol. Saarni (2000, p 68) indica además, que la competencia emocional se relaciona con la autoefiacia al expresar emociones en las transiciones sociales. Goleman, Boyatzis y Mckee (2002, p 69-73) presentan cuatro dominios: conciencia de uno mismo, autogestión, conciencia social y gestión de las relaciones. Para nosotros, la competencia emocional, en este caso adaptada a la Educación Infantil, es aquella capacidad para reconocer y expresar emociones (conciencia emocional), regular las emociones (regulación emocional), ponerse en el lugar de otro (competencia social) e identificar y resolver problemas (habilidades de vida para el bienestar).

a) Conciencia emocional

La conciencia emocional es la capacidad para tomar conciencia de las propias emociones y de las emociones de los demás, incluyendo la habilidad para captar el clima emocional de un contexto determinado (Bisquerra, 2000). Esto implica el tomar de conciencia de las propias emociones, dar nombre a las emociones y comprensión de las emociones de los demás. Por ello, es fundamental en edades tempranas, desarrollar cada uno de estos aspectos considerando el lenguaje verbal y no 
verbal como medio de expresión emocional. Tener conciencia emocional de las propias emociones es la competencia emocional fundamental sobre la que se construyen las otras (regulación, autoestima, etc.). Es decir, constituye la guía del desarrollo de las siguientes competencias.

b) Regulación emocional

Los niños han de enfrentarse a numerosas situaciones a la hora de afianzar su autonomía, aunque muchas veces no saben expresar exactamente sus deseos y necesidades. Todas estas experiencias incomodan al niño, cuyas habilidades motoras y comunicativas son todavía muy limitadas. Su autocontrol necesita desarrollarse y pueden reaccionar con rabietas, enfados, o incluso manifestar ira contra el profesor u otro alumno. El alumno de Educación Infantil debe comenzar a desarrollar la capacidad para manejar las emociones de forma apropiada, a tener buenas estrategias de afrontamiento, autogenerar emociones positivas y a desarrollar una expresión emocional apropiada. Lo cual incluye: regulación de la impulsividad (ira, violencia, comportamientos de riesgo); tolerancia a la frustración para prevenir estados emocionales negativos (ira, estrés, ansiedad, depresión); perseverar en el logro de los objetivos a pesar de las dificultades; capacidad para diferir recompensas inmediatas a favor de otras más a largo plazo pero de orden superior; estar abierto a nuevas ideas; afrontar los cambios con flexibilidad, integridad, etc.

c) Competencia social

Consisten en reconocer las emociones de los demás; saber ayudar a otras personas a sentirse bien; desarrollar la empatía, saber estar con otras personas, responder a los demás, conocer las emociones ajenas. Es una competencia que contribuye a la construcción del autoconocimiento emocional. Esta competencia permite a los alumnos de Educación Infantil a reconocer las necesidades y los deseos de otros, permitiéndoles relacionarse con los demás alumnos de forma más eficaz.

d) Habilidades de la vida para el bienestar

Se trata de ofrecer recursos que ayuden al alumno de Educación Infantil a organizar una vida sana y equilibrada, superando las posibles insatisfacciones o frustraciones. En esta competencia podemos incluir las habilidades de organización (del tiempo, del ocio, tareas cotidianas, etc.) la toma de decisiones, mantener buenas relaciones interpersonales (comunicación, cooperación, colaboración, trabajo en equipo, resolución de conflictos de la vida cotidiana, etc.), habilidades en la vida familiar, escolar y social, actitud positiva ante la vida y la percepción positiva y disfrute del bienestar. Todo ello, de cara a potenciar el bienestar personal y social y la calidad de vida del menor

\section{OBJETIVOS}

El objetivo de este estudio es analizar las competencias emocionales de niños y niñas de Educación Infantil, evaluando las posibles diferencias existentes en función del sexo. Por ello van medir y comparar las cuatro dimensiones expuestas: conciencia emocional, regulación emocional, competencia social y habilidades de vida para el bienestar..

\section{PARTICIPANTES}

La muestra la componen 123 alumnos/as $2^{\circ}$ curso de Educación Infantil escolarizados en un Centro Púbico de Educación Infantil y Educación Primaria (CEIP) de a Castilla y León, España. En función del sexo la muestra se distribuye de forma homogénea como podemos observar en la Tabla 1. 
Tabla 1.

Distribución de la muestra

\begin{tabular}{|c|c|c|c|c|c|}
\hline & \multirow[b]{2}{*}{$\mathrm{N}$} & \multicolumn{2}{|c|}{ Varones } & \multicolumn{2}{|c|}{ Mujeres } \\
\hline & & $\mathrm{N}$ & $\%$ & $\mathrm{~N}$ & $\%$ \\
\hline Muestra & 123 & 61 & 49.59 & 62 & 50.41 \\
\hline
\end{tabular}

\section{MÉTODO}

\section{Instrumentos}

\section{Escala RRER}

La Escala Reconocimiento, Regulación, Empatía y Resolución de Problemas (RRER), mide el nivel de competencia emocional, entendiendo por competencia emocional, la capacidad que tiene una persona para reconocer y expresar emociones (conciencia emocional), regular las emociones (regulación emocional), ponerse en el lugar de otro (competencia social) e identificar y resolver problemas (habilidades de vida para el bienestar).

Las investigadoras del presente estudio desarrollaron un instrumento que evalúa el desarrollo emocional a través de los siguientes componentes: conciencia emocional, regulación emocional, competencia social y habilidades de vida para el bienestar. Este instrumento utiliza láminas evocadoras de situaciones apoyadas por preguntas del examinador o examinadora. En total la prueba cuenta con 65 ítems. La prueba alcanzó en su puntaje total un Alfa de Cronbach de 0.865, en conciencia emocional 0.73 , en regulación emocional 0.705 y en competencia social 0.758 y en habilidades de vida para el bienestar 0.760 .

\section{Procedimiento}

La escala RRER se aplicó individualmente a cada uno de los menores que componen la muestra, dedicando entre 15 y 25 minutos por niño/a. Posteriormente se procedió a informatizar y codificar los datos en el programa Statistical Package for the Social Sciences 21 (SPSS; versión 21). Los análisis realizados consintieron principalmente en pruebas no paramétricas para muestras independientes, a fin de comparar las puntaciones de los niños y de las niñas en las cuatro dimensiones señaladas.

\section{Resultados}

En primer lugar, nos vamos a centrar en el análisis estadístico descriptivo del nivel de competencias emocionales. Para toda la muestra, las puntuaciones oscilan entre 13 y 59, la media se sitúa en el valor de 42,12 con una desviación típica de 8,60 (Tabla 2). Como podemos observar en la Tabla 2, las puntuaciones de las niñas son más elevadas que las de sus compañeros varones, siendo mayor incluso la puntuación mínima.

En la Tabla 3, se presenta la comparación de varones y mujeres en su puntuación en las escala RRER. Como ya habíamos anticipado, las niñas tienen un nivel global de competencia emocional mayor que sus compañeros, extiendo diferencias significativas entre ambos grupos $(p=.001)$.

Comparando de forma desglosada las puntuaciones de niños y de niñas en las dimensiones que conforman la competencia emocional encontramos diferencias significativas en tres de ellas. Como podemos observar en la Tabla 4 las mujeres obtienen puntuaciones más elevadas en todas las dimensiones. En el caso de la regulación emocional la diferencia es significativa $(p=.011)$, es decir, 
Tabla 2.

Estadísticos descriptivos de las competencias emocionales en función del sexo

\begin{tabular}{|c|c|c|c|c|c|c|c|c|}
\hline & \multicolumn{4}{|c|}{ Puntuación } & \multirow[b]{2}{*}{ DT } & \multicolumn{3}{|c|}{ Percentil } \\
\hline & $\mathrm{n}$ & mín. & máx. & media & & Q1 & Q2 & Q3 \\
\hline Muestra & 123 & 13 & 59 & 42.12 & 8.60 & 36 & 43 & 49 \\
\hline Mujeres & 62 & 28 & 59 & 44.74 & 7.04 & 40 & 46 & 50 \\
\hline Varones & 61 & 13 & 54 & 39.45 & 9.25 & 34 & 41 & 46 \\
\hline
\end{tabular}

Tabla 3.

Diferencias en función del sexo

\begin{tabular}{lrrrrc}
\hline & \multicolumn{1}{c}{$\begin{array}{c}\text { Rango } \\
\text { promedio }\end{array}$} & $\begin{array}{c}\text { Suma de } \\
\text { rangos }\end{array}$ & \multicolumn{1}{c}{$\mathrm{Z}$} & $\mathrm{p}$ \\
\cline { 2 - 6 } Mujeres & 62 & 72.20 & 4476.50 & & \\
Varones & 61 & 51.63 & 3149.50 & -3.20 & .001 \\
\hline
\end{tabular}

Tabla 4.

Dimensiones de las competencias emocionales en función del sexo

\begin{tabular}{|c|c|c|c|c|c|}
\hline & $\mathrm{N}$ & $\begin{array}{l}\text { Rango } \\
\text { promedio }\end{array}$ & $\begin{array}{l}\text { Suma de } \\
\text { rangos }\end{array}$ & Z & $\begin{array}{l}\text { Sig.asintót. } \\
\text { (bilateral) }\end{array}$ \\
\hline \multicolumn{6}{|c|}{ Conciencia emocional } \\
\hline Mujeres & 62 & 66.40 & 4147 & \multirow{2}{*}{-1.38} & \multirow{2}{*}{.165} \\
\hline Varones & 61 & 57.52 & 3509 & & \\
\hline \multicolumn{6}{|c|}{ Regulación emocional } \\
\hline Mujeres & 62 & 68.81 & 4266 & \multirow{2}{*}{-2.52} & \multirow{2}{*}{.011} \\
\hline Varones & 59 & 52.80 & 3115 & & \\
\hline \multicolumn{6}{|c|}{ Competencia social } \\
\hline Mujeres & 62 & 71.79 & 4451 & \multirow{2}{*}{-3.10} & \multirow{2}{*}{.002} \\
\hline Varones & 61 & 52.05 & 3175 & & \\
\hline \multicolumn{6}{|c|}{$\begin{array}{l}\text { Habilidades de vida para el } \\
\text { bienestar }\end{array}$} \\
\hline Mujeres & 62 & 68.98 & 4277 & \multirow{2}{*}{-2.22} & \multirow{2}{*}{.026} \\
\hline Varones & 61 & 54.90 & 3349 & & \\
\hline
\end{tabular}


las niñas regulan mejor sus respuestas emocionales. En la dimensión de competencia social, nuevamente encontramos diferencias significativas entre chicos y chicas $(p=.002)$, siendo estas últimas quienes tienen una mayor competencia social. En consonancia con estos resultados, encontramos nuevamente diferencias significativas entre varones y mujeres $(p=.026)$ en las habilidades de vida para el bienestar. Las niñas también obtienen una puntuación más alta en esta dimensión.

\section{CONCLUSIONES}

Esta investigación se ha centrado en el estudio de las competencias emocionales del alumnado de Educación Infantil y en la evaluación de las posibles diferencias en el desarrollo emocional de niños y niñas. Pese a los obstáculos existentes para la medición y valoración de las competencias emocionales de los menores, consideramos que la escala "RRER" es un instrumento útil para esta tarea.

Los resultados obtenidos sugieren que las niñas poseen un mayor desarrollo emocional en relación a sus compañeros varones, siendo éste un aspecto controvertido en la investigación previa. Respecto a la regulación emocional, donde nosotros si encontramos niveles superiores en niñas, existen perspectivas contradictorias. Por un lado, los trabajos de Joseph y Newman (2010) 0 Salovey (2006), coinciden con nuestra investigación, encontrando en varones peores patrones de regulación emocional. Otras investigaciones sin embargo, encuentran en varones puntuaciones superiores en regulación emocional (Bar-On, 2006; Sánchez, Fernández-Berrocal, Montañés, y Latorre, 2008). En cuanto a la competencia social, principalmente la empatía, nuestros resultados coinciden con las investigaciones previas dado que las niñas demuestran mayor empatía (Garaigordobil y García, 2006; Mestre, Samper, Frias y Tur 2009; Sánchez-Queija, Oliva y Parra, 2006). No obstante, debemos ser prudentes pues los menores de estos trabajos tienen mayor edad que los sujetos de nuestra muestra. En cualquier caso, creemos que es necesario seguir indagando sobre la relación del sexo en las competencias emocionales, planteándose la influencia de las exceptivas sociales en estos resultados. Por ello, se torna fundamental la investigación en los primeros años de vida, así como el análisis de la misma desde una perspectiva de género.

Los resultados obtenidos avalan la necearía implementación de la Educación Emocional en los currículos escolares. El aprendizaje de las competencias emocionales, tanto en niños como en niñas, debe convertirse en una prioridad en el sistema educativo. Centrándonos en la etapa de la Educación Infantil, donde se han de ofrecer las primeras experiencias educativas, es imprescindible ofrecer talleres y tareas donde se movilicen las competencias emocionales, dada su importancia social y formativa. Además, sostenemos que este "viaje hacia la educación emocional" sólo es posible con la implicación de todos los agentes educativos: familias, profesores, tutores, etc.

\section{REFERENCIAS BIBLIOGRÁFICAS}

AGULLÓ, M.J., FILELLA, G., SOLDEVILA, A., Y RIBES, R. (2011). Evaluación de la educación emocional en el ciclo medio de Educación Primaria. Revista de educación, 354, 347-349.

AMBRONA, T., LÓPEZ-PÉREZ, B., y MÁRQUEZ-GONZÁLEZ, M. (2012). Eficacia de un programa de eduación emocional breve para incrementar la competencia emocional de niños de educación primaria. Revista Española de Orientación y Psicopedagogía (REOP), 23(1), 39-49.

BISQUERRA, R. (2000). Educación emocional y bienestar. Barcelona: Praxis.

DOBRIN, N Y KÁLLAY, E. (2013). The Investigation or the Short-Term Effects of a Primary Prevention Program Targeting the Development of Emotional and Social Competencies in Preschoolers. Cognitie, Creier, Comportament, 17(1), 15-34.

FERNÁNDEZ-BERROCAL, P., Y EXTREMERA, N. (2009). La Inteligencia Emocional y el estudio de la felicidad. Revista interuniversitaria de formación del profesorado, 66, 85-108. 
GARAIGORDOBIL, M. Y GARCÍA, P. (2006). Empatía en niños de $10^{\underline{a}} 12$ años. Psicothema, 18(2), 180-186.

GOLEMAN, D., BOYATZIS, R., Y MCKEE, A. (2002). Primal leadership: Realizing the power of emotional intelligence. Boston, MA, USA: Harvard Business Scholl Press.

JOSEPH, D. L., Y NEWMAN, D. A. (2010). Emotional Intelligence: An Integrative Meta-Analysis and Cascading Model. Journal of Applied Psychology, 95(1), 54-78.

LAU, P Y WU, F. (2012). Emotional competence as a positive youth development construct: a conceptual review. The Scientific World Journal, 2012. Doi: 10.1100/2012/975189.

MESTRE, V., SAMPER, P., Y FRÍAS, M.D. (2002). Procesos cognitivos y emocionales predictores de la conducta prosocial y agresiva: la empatía como factor modulador. Psicothema, 14(2), 227232.

SAARNI, C. (1999). The development of emotional competence. Nueva York: Guilford.

SALMERON, P. (2002). Evolución de los conceptos, sobre Inteligencia Emocional para la orientación. Educación XX. Revista de la Facultad de Educación, 5, 98-121.

SALOVEY, P. (2006). Epilogue: The Agenda for Future Research. En V. DRUSKAT, F. SALA, Y G. MOUNT (Eds.), Linking El and Performance at Work Current Research Evidence with Individuals and Groups (pp. 267-272). LEA Inc.

SALOVEY, P., Y GREWAL, D. (2005). The science of emotional intelligence. Current Directions in Psychological Science, 14, 281-285.

SALOVEY, P. Y SLUYTER, D. J. (1997). Emotional Development and Emotional Intelligence. Educacional Implications. Nueva York: Basic Boocks.

SÁNCHEZ- QUEIJA, I., OLIVA, A. Y PARRA, A. (2006). Empatía y conducta prosocial durante la adolescencia. Revista de Psicología Social, 21 (3), 259-271.

SÁNCHEZ, M.T., FERNÁNDEZ-BERROCAL, P., MONTAÑES, J., Y LATORRE, J.M. (2008) ¿Es la inteligencia emocional una cuestión de género? Socialización de las competencias emocionales en hombres y mujeres y sus implicancias. Revista Electrónica de Investigación Psicoeducativa, 6(2) 455-474.

TERUEL, P. (2014). Por qué y para qué la Educación Emocional. Cuadernos de pedagogía, 442, 6264. 
